\title{
Enhanced extraction of an anticancer drug, Vinblastine, from Catharanthus roseus
}

Sumera Javad*, Sumera Sarwar, Khajista Jabeen, Sumera Iqbal and Amina Tariq

Department of Botany, Lahore College for Women University, Lahore, Pakistan

*Corresponding author's email: zif 4@yahoo.com

Citation

Sumera Javad, Sumera Sarwar, Khajista Jabeen, Sumera Iqbal and Amina Tariq. Enhanced extraction of an anticancer drug, Vinblastine, from Catharanthus roseus. Pure and Applied Biology. Vol. 5, Issue 3, pp608-614. http://dx.doi.org/10.19045/bspab.2016.50079

Received: 07/02/2016

Revised: 03/06/2016

Accepted: 08/06/2016

Online First: 21/06/2016

\section{Abstract}

Catharanthus roseus G. Don belonging to family Apocyanaceae, contains more than 200 important compounds among which Vinblastine is an important anti-cancerous alkaloid. An efficient method for its extraction from plant material can lower its cost internationally. Study was aimed to increase the efficiency of extraction for the anticancer drug Vinblastine from C. roseus. In present study a comparison was made between different extractions methods for Vinblastine i.e., Microwave Assisted Extraction (MAE) and Soxhlet Extraction using a few drops of conc. $\mathrm{HCl}$ and ethanol respectively as extracting solvent. Quantitative estimation of Vinblastine was done with the help of HPLC (High performance liquid chromatography). Acetonitrile was used as a mobile phase. It was proved that microwave assisted extraction is more effective and efficient for extraction of Vinblastine. Microwave assisted extraction of leaves of C. roseus produced a maximum of $0.43 \mathrm{~g}$ of extract per gram of plant material while using lesser time of extraction (30 seconds) with a very little amount of solvent used $(10 \mathrm{~mL} / \mathrm{g})$ as compared to Soxhlet extraction (19.01g/30 g of plant material) and extraction time(10 hours). The maximum concentration of Vinblastine was found to be $44.33 \mathrm{mg} / \mathrm{g}$ of plant sample at 60 Seconds of microwave assisted extraction of power level 700W by using High Performance Liquid Chromatography (HPLC). Hence it is concluded that Microwave assisted extraction is a rapid and efficient tool for extraction of Vinblastine from $C$. roseus.

Key words: High Performance Liquid Chromatography (HPLC); HCl; Microwave Assisted Extraction (MAE); Soxhlet extraction; Alkaloid

\section{Introduction}

Cancer is an abnormal growth and rapid increase in the amount of cells. There are over 200 different known cancers that affect humans. In chemotherapy, a number of chemicals are in use which have anticancerous activities and are used to cure tumors. But these have very severe side effects due to which they have limited usage [1]. The search for anticancer agent from plant sources started in 1950 with the discovery and development of Vinca rosea alkaloid, Vinblastine and Vincristine [2]. All plants have antioxidants in them. This is a gift 
of nature, so plants show anticancer activity. Plants with larger amounts of antioxidants show increased activity against cancers with no or very less toxicity or side effects because they are natural products as compared to synthetic products or modern allopathic products.

C. roseus (L.) is a highly specialized plant for the biosynthesis of different monoterpenoidindol alkaloids (MIAs), and many of them have strong biological activities [3]. C. roseus (L.) belongs to family Apocyanaceae. It is also called myrtle. It is an ever green herb or shrub and a perennial plant occurring in West Indies and Madagascar and also cultivated in India in large amount. This plant contains more than 200 important compounds (alkaloids). In these alkaloids one is Vinblastine that is widely used in medicines. Study also shows that low concentration of Vinblastine $(0.1$ $6 \mathrm{nM})$ inhibits the development of mitosis from metaphase to anaphase in HeLa cells without causing net depolymerization of microtubules $[4,5]$.

Extraction, the basic step for getting herbal product, is the separation of a substance from a matrix. There are many methods that are used for extraction purpose. Heat reflux extraction, Liquid-liquid extraction, soxhlet extraction, Solid phase extraction, ultrasonic extraction, Supercritical carbon dioxide extraction and microwave-assisted extraction methods are also used for extraction purposes [6]. In modern herbal industry, modern methods of extraction have played their role in number of ways i.e., increasing the stability of final product, decreasing the use of hazardous solvents and eliminating the unwanted components of extract as well as decreasing the time and energy consumption $[7,8]$. Microwave assisted extraction is one of these modern methods which is in fact a combination of modern technology and conventional solvent extraction. HPLC is an analytical technique often accompanied by extraction methods to validate the protocol. It gives the more accurate estimation of the quantity of molecule of interest. It can provide validated information about product recovery and purification level even at an industrial scale [9].

In present study a comparison is made between soxhlet extraction and microwave assisted extraction of Vinblastine from $C$. roseus by applying HPLC analysis.

\section{Materials and methods}

Leaves of $C$. roseus were collected from surroundings of Lahore District Pakistan, dried under shade and were finely ground. For MAE accurately weighed $1 \mathrm{~g}$ of the $C$. roseus powder was placed in vial each time. Measured quantity of solvent $(10 \mathrm{ml})$ was added in beaker along with $1 \mathrm{~g}$ plant material and $0.2 \mathrm{ml}$ of $\mathrm{HCl}$; and was covered with polythene bags to avoid solvent evaporation. Different power levels were used for maximum extraction of alkaloids. i.e., 500, 700 and $900 \mathrm{~W}$. Time series ranged from 3090 seconds. Ethanol and water were used separately for extraction of alkaloids. The extract was placed in pre-weighed vials each time and placed under fan for drying. Dry extract was stored in fridge at $4^{\circ} \mathrm{C}$. For Soxhlet extraction 30 grams of $C$. roseus leaves in powdered form were loaded in extracting unit of soxhlet, it was done carefully to ensure the maximum possible contact of plant matrix and solvent. The range selected for time of extraction was 515 hours and ethanol was used as a solvent. The final extract was evaporated by rotary evaporator at first then it was placed in preweighed china dishes to be dried completely. Dry extract thus obtained was placed at $4^{\circ} \mathrm{C}$ in refrigerator. Extracts were weighed accurately each time and data was recorded carefully.

Further quantification of Vinblastine was done by HPLC equipment (Shimardzu, Japan). An ODS-C18 column (4.6 x250 mm) with pore size $5 \mu \mathrm{m}$ was used. A UV-Visible 
detector was attached with, and was set at $254 \mathrm{~nm}$. Standard Vinblastine was prepared by dissolving $0.1 \mathrm{mg}$ of powder in $10 \mathrm{ml}$ of solvent and $20 \mu \mathrm{l}$ of it was injected into the equipment. All the extract solutions were prepared for HPLC analysis by mixing 0.1 mg of extract in $10 \mathrm{ml}$ of solvent and were filtered separately with micro-filters of $0.45 \mu$ pore size. Quantitative estimation for Vinblastine was made with the help of formula:

Percentage of Vinblastine $=\frac{W_{s t} \times A_{a}}{W_{a} \times A_{s t}} \times 100$

Where $W_{\text {st }}$ represents weight of standard compound, $\mathrm{W}_{\mathrm{a}}$ shows the weight of sample extract, $A_{a}$ shows Peak area of sample compound and $A_{s t}$ is peak area of standard compound.

\section{Results}

A comparison was made between different extractions methods for anticancer drug Vinblastine from $C$. roseus. Extraction was carried out by using two different methods i.e., MAE and Soxhlet Extraction using a few drops of conc. $\mathrm{HCl}$ and ethanol respectively as extracting solvent. Estimation of Vinblastine was done by using HPLC. Table 1 shows the effect of time and power level of microwaves on yield of extract from dry leaf powder of $C$. roseus. The maximum amount of extraction was at 30 seconds at power level of $900 \mathrm{~W}(0.43 \mathrm{mg}$ per $\mathrm{g}$ of leaf powder) while minimum amount of extract was $0.16 \mathrm{~g}$ per gram of leaf powder at 90 seconds at same power level. On increasing the power level from 700 to $900 \mathrm{~W}$ amount of extract started decreasing. Table 2 depicts the amount of extract by using the soxhlet extraction. It is clear that the maximum amount of extract obtained was $19.01 \mathrm{mg}$ per 30 gram of plant material after 15 hours of extraction.

Figure 1 relates the amount of Vinblastine (per gram of plant material estimated by HPLC) with the amount of extracts by using microwave assisted extraction and soxhlet extraction. It shows that the amount of Vinblastine is very much higher in microwave assisted extracts as compared to soxhlet extracts. Highest amount of Vinblastine was estimated $(44.33 \mathrm{mg} / \mathrm{g}$ of plant sample) in microwave assisted extract at $700 \mathrm{~W}$ and 60 seconds of microwave irradiation (Figure 2). While on increasing or decreasing the time of irradiation at this power level, amount of Vinblastine decreased (Figure 3 ). A very little amount of Vinblastine is extracted by using soxhlet extraction (Figure 1 and 4). Peak of standard Vinblastine is depicted in Figure 5.

Table 1. Effect of time and power of microwaves on extraction of $C$. roseus leaves using MAE

\begin{tabular}{|c|c|c|c|}
\hline Sr. \# & Power level (W) & Time of Extraction (Sec) & Weight of extract \\
\hline 1 & 500 & 30 & $0.39^{\mathrm{a}} \pm 0.005$ \\
2 & & 60 & $0.31^{\mathrm{b}} \pm 0.003$ \\
3 & & 90 & $0.28^{\mathrm{b}} \pm 0.032$ \\
\hline 4 & 700 & 30 & $0.31^{\mathrm{a}} \pm 0.012$ \\
5 & & 60 & $0.25^{\mathrm{b}} \pm 0.037$ \\
6 & & 90 & $0.17^{\mathrm{c}} \pm 0.020$ \\
\hline 7 & 900 & 30 & $0.43^{\mathrm{a}} \pm 0.008$ \\
8 & & 60 & $0.20^{\mathrm{b}}+0.053$ \\
9 & & 90 & $0.16^{\mathrm{c}} \pm 0.044$ \\
\hline
\end{tabular}

Each value is the mean of three replicate with standard error (mean \pm S.E). Means within a column not sharing a common superscript differ significantly $(\mathrm{P}<0.05)$ according to Duncan's new multiple range test. 
Table 2. Effect of time on extraction yield of $C$. roseus leaves by using Soxhlet

\begin{tabular}{|c|c|c|}
\hline Sr. \# & Time of extraction & Weight of extract \\
\hline 1 & 5 hours & $10.03^{\mathrm{c}} \pm 0.019$ \\
\hline 2 & 10 hours & $19.01^{\mathrm{a}} \pm 0.020$ \\
\hline 3 & 15 hours & $18.03^{\mathrm{b}} \pm 0.028$ \\
\hline
\end{tabular}

Each value is the mean of three replicate with standard error (mean \pm S.E). Means within a column not sharing a common superscript differ significantly $(\mathrm{P}<0.05)$ according to Duncan's new multiple range test.

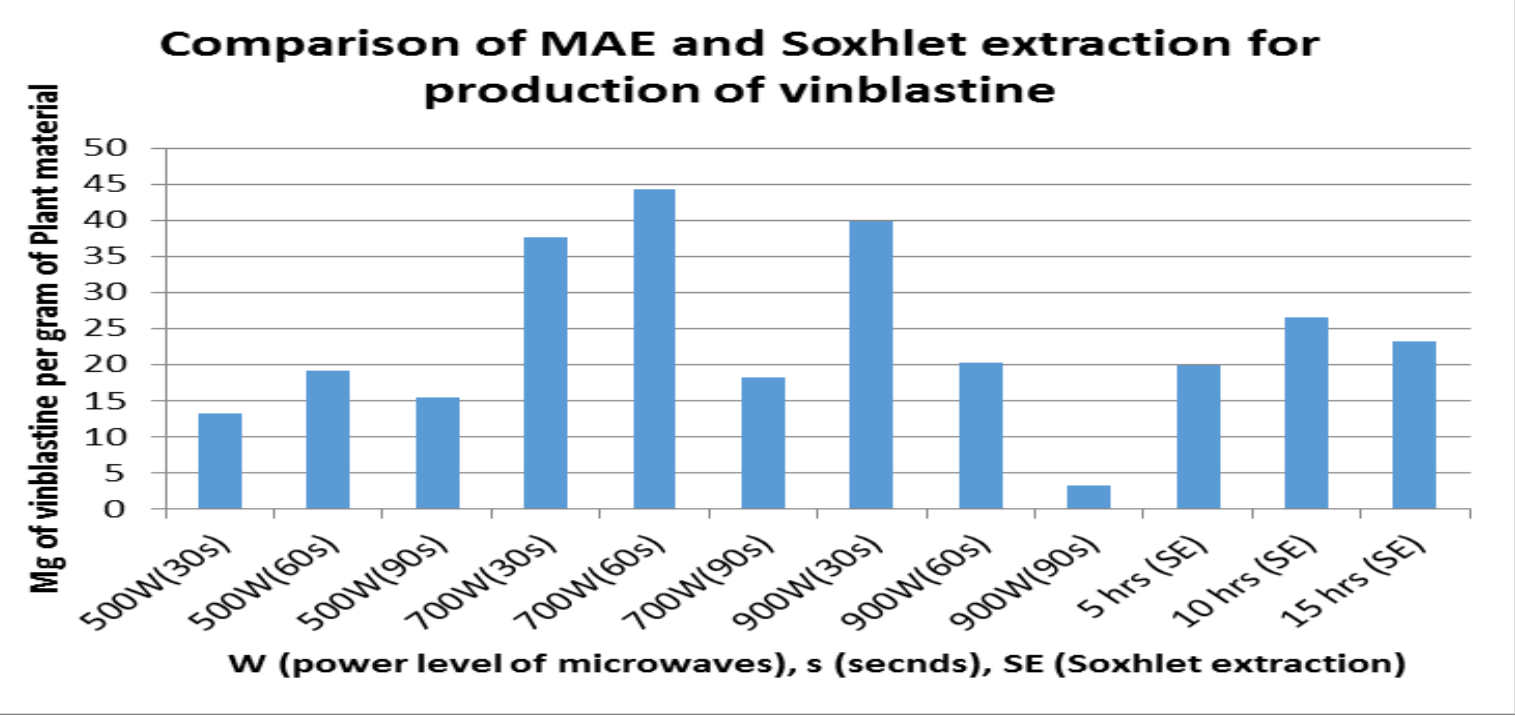

Figure 1. Comparison of MAE and Soxhlet extraction for the extraction of Vinblastine

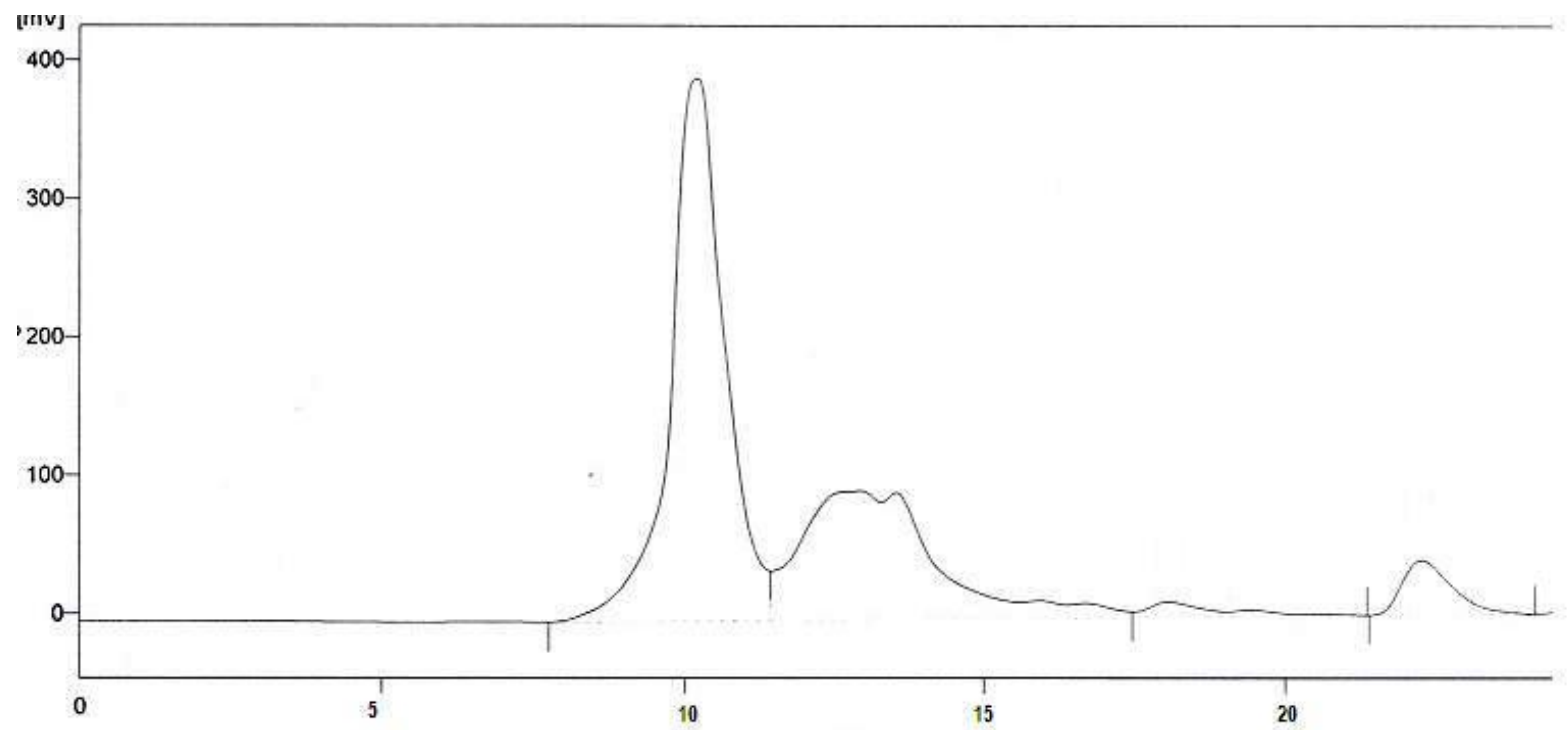

Figure 2. HPLC chromatogram showing amount of Vinblastine at $700 \mathrm{~W}$ and 60 seconds of microwave assisted extraction 


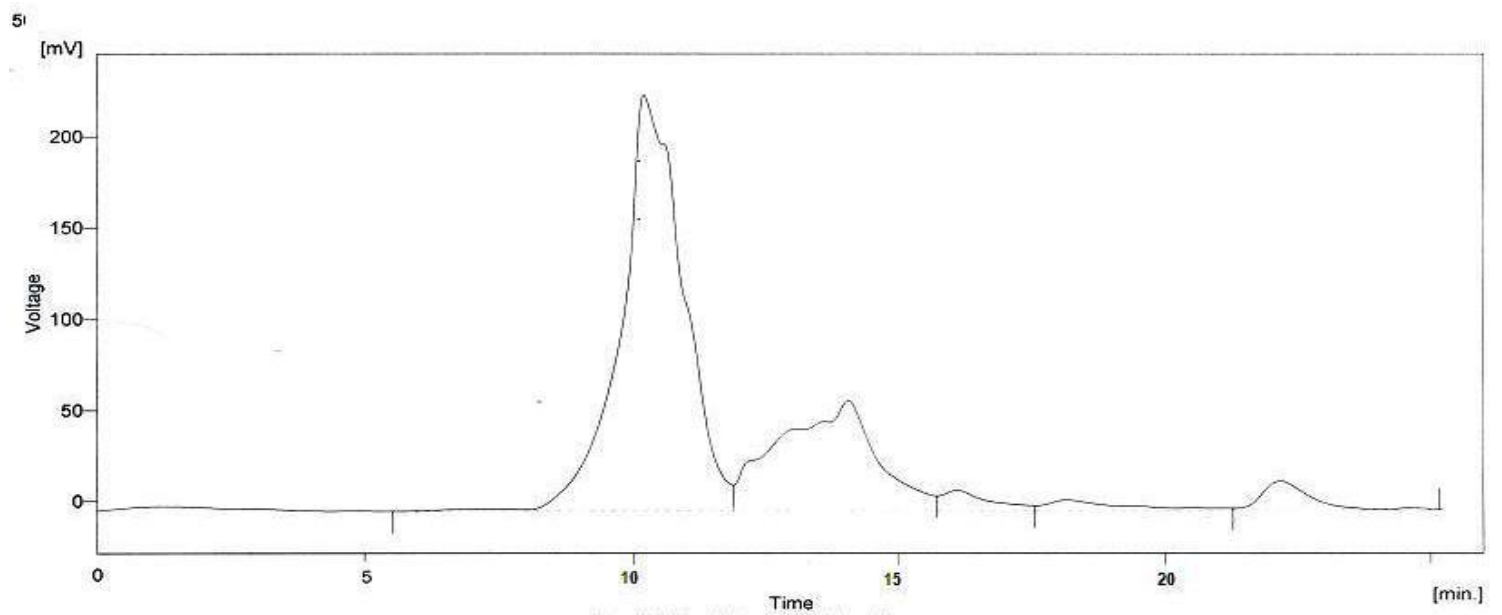

Figure 3. HPLC chromatogram showing amount of Vinblastine at $700 \mathrm{~W}$ and 90 seconds of microwave assisted extraction

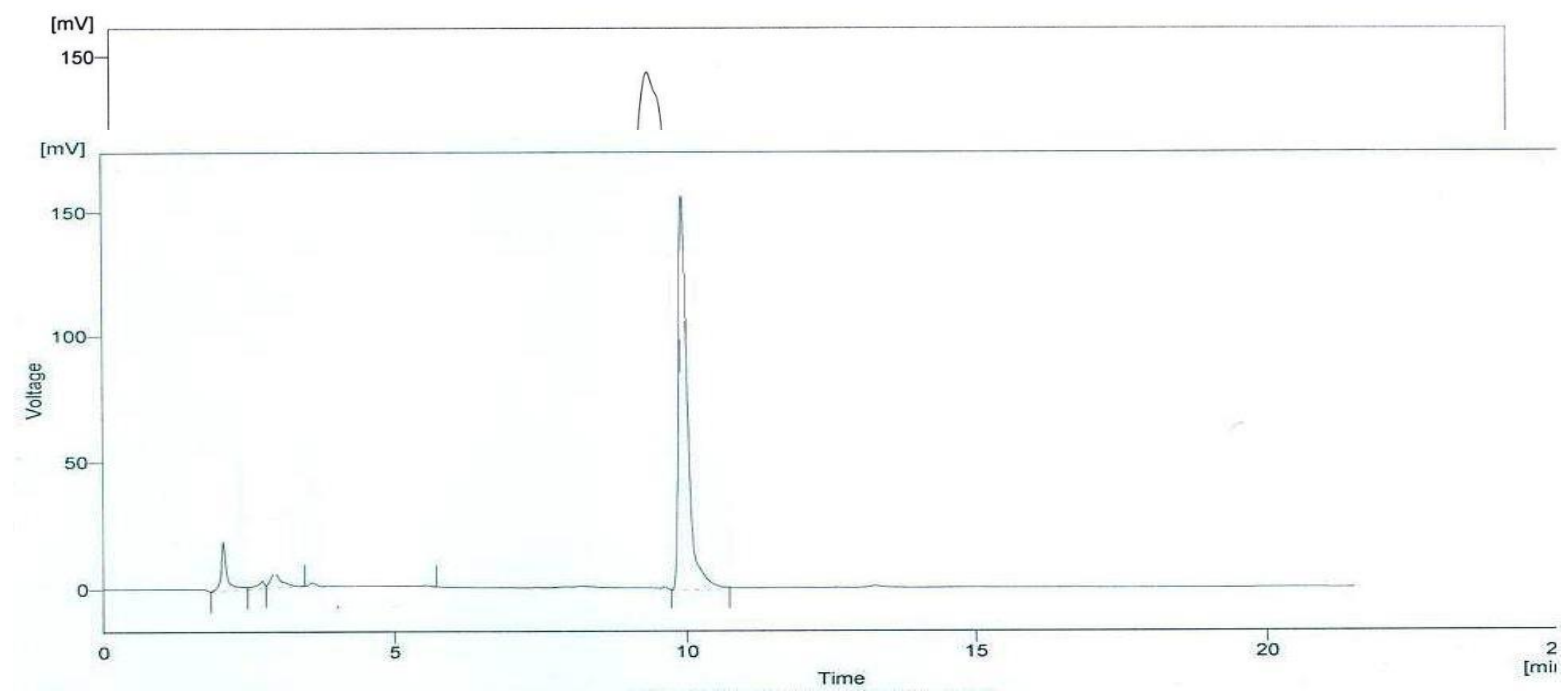

Figure 4. HPLC chromatogram showing amount of Vinblastine after 15 hours of soxhlet extraction

Figure 5. HPLC chromatogram of standard Vinblastine

\section{Discussion}

At present there is an increasing trend of researchers to optimize such methods of extractions for herbal products which are automated, use lesser solvents, consume lesser energy are particularly environment friendly. When different methods of extraction are considered, the common thing in them is elevated temperatures or long durations of extractions. While in all modern methods lesser time of extraction as well as lesser solvent use, make them preferable over conventional extraction methods. Among all these extraction methods, microwave assisted extraction is very significant. In this method sample is rapidly heated due to number of reasons. For example in conventional method, at first vessel containing the sample is heated and then heat is conducted to the plant sample while in microwave assisted extraction plant sample is directly heated [10]. Furthermore microwaves cause the 
breaking of $\mathrm{H}$-bonds of cell wall and membranes of cells which in turn enhances the extraction rate. But very high power levels with higher exposure times penetrate into the plant metabolite structures and cause breakage of hydrogen bonds there. In MAE, by targeting the different parameters like temperature, power level, time of extraction and solvent type; quality and quantity of target compound can be controlled. In this way a pure product can be obtained, due to which there is no need of intermediate purification steps and cost is also reduced [11].This explains the enhanced extraction of Vinblastine by microwave assisted extraction. This is the main requirement for the economical commercial production of Vinblastine against increasing threat of cancer.

In present study a comparison was made between microwave assisted and soxhlet extraction of Vinblastine from $C$. roseus and it was clear from results that higher content of anticancer drug-Vinblastine was obtained in lesser time by MAE. Earlier Pan et al., (2002) explained the advantage of MAE in its comparison with the conventional extraction techniques for the extraction of tanshinones from Salvia miltiorrhiza bunge [12]. While Javad and coworkers reported the lesser time of extraction of stevioside by MAE from Stevia rebaudiana Bertoni as compared to soxhlet extraction [13]. In soxhlet extraction, sample is not agitated so mixing and contact of solvent and plant matrix is not perfect, due to which more time is required to extract more. Agitation is basically responsible for rapid bond breaking of plant matrix and releasing target compounds [14].

Rydberg and Reinhardt, reported that maximum amount of extraction in $C$. roseus was found by soxhlet extraction. They used $17 \mathrm{~g}$ of air dried plant material for the extraction with $50 \mathrm{ml}$ of hexane which took almost 6 hours for maximum extraction but the time taken ( 6 hours) is far more than the targeted extraction of Vinblastine in the present study (30 seconds). Time of irradiation and power level of microwaves are important correlated factors which in fact strongly affect the final yield of plant secondary metabolites. As in any extraction experiment, time of extraction determines the bond breakage duration and final release of the product. Low power of irradiation with long exposure is usually preferable over extractions with high power of irradiation with less exposure as it may cause degradation of the target compound, explosion due to overheating of the solvent or solvent losses [15, 16]. Moreover lower power levels cause the slow cell wall destruction and gradual release of exudates so making microwave assisted extraction more selective for the target compound [17]. Microwaves are used for the extraction of compounds of commercial importance in less time, with lesser solvent and causing comparatively lesser harm to the environment [18].

\section{Authors' contributions}

Research idea and design and supervised the work: S Javad, Research work of extraction: S Sarwar, Statistical analysis: K Jabeen, HPLC analysis: S IIqbal, Wrote the paper: A TTariq.

\section{References}

1. Gupta AK \& Tandon N (2004). Reviews on Indian Medicinal Plants. J Med Plants Stud 2: 119-129.

2. Cragg GM \& Newman DJ (2009). Natural product scaffolds as leads to drugs. Future Medicinal Chemistry 1(8): 1415-1427.

3. Yuan F, Wang Q \& Pan Q (2011). An efficient somatic embryogenesis based plant regeneration from the hypocotyls of $C$. roseus. An efficient somatic embryogenesis based plant regeneration from the hypocotyls of C. roseus. Afr J Biotech 10(66): 14786-14795.

4. Jordan MM, Thrower D \& Wilson L (1991). Mechanism of inhibition of cell 
proliferation by Vinca alkaloid. $J$ Cancer Res 51(8): 2212-2222.

5. Jordan MM, Thrower D \& Wilson L (1992). Effect of vinblastine, podophyllotoxin and nocodazole on mitotic spindle, complication for the role of microtubule dynamics in mitosis. $J$ Cancer Res 102: 401-416.

6. Pan X, Guoguang N \& Huizhou L (2003). Microwave-assisted extraction of tea polyphones and tea caffeine from green tea leaves. Chem. Eng. and Process 42(2): 129-133.

7. Gupta A, Naraniwal $M$ \& Kothari V (2012). Modern extraction methods for preparation of bioactive plant extracts. Int. J. Appl. Nat. Sci 1(1): 250-254.

8. Delazar A, Nahar L \& Hamedeyazdan S (2012). Microwave-assisted extraction in natural products isolation. J. Anal. Chem 864: 89-115.

9. Zhou ML, Shao JR \& Tang YX (2009). Production and metabolic engineering of terpenoidindole alkaloids in cell cultures of the medicinal plant $C$. roseus (L.) (Madagascar periwinkle). J Biotech Appl Biochem 2: 313-323.

10. Letellier M \& Budzinsk H (1999). Microwave assisted extraction of organic compounds. Analysis 27: 259271.

11. Eskilsson CS, Erland B (2000). Analytical-scale microwave-assisted extraction. J Chrom A 902: 227-250.

12. Pan X, Niu G \& Liu H (2002). Comparison of microwave-assisted extraction and conventional extraction techniques for the extraction of tanshinones from Salvia miltiorrhiza Bunge. Biochem Eng J 12(1): 71-77.

13. Javad S, Shagufta N, Saiqa I, Amna T \& Farah A (2014). Optimization of the Microwave assisted extraction and its comparison with different conventional extraction methods for isolation of stevioside from Stevia rebaudiana. Asian J Chem 26(23): 8043-8048.

14. Eljarrat E, Caixach J \& Rivera J (1998). Microwaves Vs soxhlet for the extraction of PCDDs and PCDFs from sewage sludge samples. Chemosphere 36(10): 2359-2366.

15. Rydberg J \& Reinhardt H (2000). Centrifuge for complete phase separation of two liquids. U.S. Patent 3: 442-445.

16. Raman G \& Gaikar VG (2002).Extraction of piperine from Piper nigrum (black pepper) by hydrotropic solubilization. Indus \& Eng Chem Res 41(12): 2966-2976.

17. Zhang Y, Giboulot A \& Zivy M (2011). Combining various strategies to increase the coverage of the plant cell wall glycoproteome. Phytochem 72: 11091123.

18. Soylak M, Tuzen M \& Souza AM (2007). Optimization of microwave assisted digestion procedure for the determination of Zinc, Copper and Nickel in tea samples employing flame atomic absorption spectrometry. $J \mathrm{Haz}$ Mat 149(2): 264-268. 